Sanguinetti S. y cois.

Rev. Chill. Pediatr. 64 (2); 94-99, 1993

\title{
Rendimiento escolar en niños nacidos con bajo peso
}

\author{
Sylvia Sanguinetti T.'; Erika Koplin I. ; Jorge Torres P. ${ }^{3}$
}

Birth weight and school performance

The influence of low birthweight, weighl adequacy to gestolional age and gestational age on learning at elementary school was sludied in two groups of 82 children each, aged $710 \% 121088 / 12$ ycors, all born in Arico's regional hospital at northern Chile in 1980. Children of the first group up had low birthweights, while those of the second one weighed more than $2500 \mathrm{~g}$ at birth, but bolh groups wese similat in sccioeconomic aspects and gender distribution. After dismissal of four subjecls in each' group becouse of clearcut mental deficiency, low birthweight children in: 78! had significontly lower achievemenl in mean reoding 153.07 vs $61.91, p<0.001$, witing $[12.23 \mathrm{vs} 13.68,0<0.05$, errors in reading $[54.22$ \& $00.27, p<0.05\}$ and leclure comprehension $[42.04$ v $48.58, p<0.001\}$ scores. Children born premalurely in: 50 ) hod significantly lower levels of achievement in reading $\{53.88$ vs $59.9, p<0.05$ ) and errors in reading $[53.78$ vs $58.88,0<0.05$ ) Ihan childien who were fultterm babies (n: 100 ). Those who were small for gestational age in: 59 ) showed grealer development of visual molor integration $\{1$ ! I.49 vs $100.72,0<0,05\}$ than the group who were adequale $[\mathrm{m}$ : 97]. These resulis support the need of preventive educalion programs for thesc high-risk gloups.

(Koy words: learning, learning disorders, birthweight.]

El bajo peso de nacimiento (BPN) es una condición de riesgo para cl niño, pues está asociado a la mayoría de las defunciones neonatales ${ }^{1}$. En la medida que los avances en las atenciones y cuidados intensivos han permitido disminuir la mortalidad neonatal ${ }^{2.3}$ se hace necesario evaluar la calidad de vida de los sobrevivientes. En los últimos años diversos estudios de seguimiento cn el extranjero muestran una disminución significativa de las secuelas neurológicas y sensoriales más severas ${ }^{4}$. No obstante, estos nifios, especialmente aquellos de muy bajo peso de nacimiento, presentan más frecuentemente limitaciones intelectuales que la población general ${ }^{5-8}$. Las alteraciones menos severas no se advierten durante los primeros años de vida, sino que son más manificstas en periodos posteriores en los cuales

1. Magíster en Educación, Centro de Diagnossico, A rica,

2. Psicologa. Escueja de Psicología, Pontificia Universidad Católica de Chile.

3. Médico, Unidad de Recién Nacidos, Hospital San BerjaArriarán.

Trabajo realizado con la colaboración del Programs de Salud Perinatal - Fundación W.K. Kellogg el niño requiere integrar funciones y poner en práclica mecanismos más complejos de adaplación, tales como los requeridos para un buen desempeño escolar $6,9-13$.

Esle estudio tiene como objetivo determinar la influencia del bajo peso de nacimiento sobre algunos aspectos del aprendizaje escolar en niffos nacidos con esta característica en Arica en 1980. Asimismo, se analizaron la prematurez y la adecuación a la edad gestacional, condiciones asociadas a la posibilidad de presentar trastornos en el desempefio escolar ${ }^{14}$. La importancia de precisar el impacto del bajo peso de nacimiento en el rendimiento escolar, reside en su potencial para contribuir a justificar la aplicación de medidas de apoyo y rehabilitación en esta área.

\section{Material y Método}

Del total de 3489 nacidos vivos en el hospital Juan Nioé, de Arica, durante el año 1980, 168 fueron de BPN (5\%), de los cuales 50 murieron, 47 en el periodo neonatal y 3 pos. teriormente. Cabc hacer nolar que en esa época no había unidad de cuidados intensivos neonatales en el hospital، lo que explicaría la elevada mortalidad neonatal (14,3\%). De los 118 sobrevivientes se excluyeron 36 nifos por cambio 
de residencia, vivir en zonas rurales de difícil acceso o tener antecedentes mćdicos incompleıs. De esta manera el grupo de estudio quedó constituido funalmente por 82 niños nacidos con peso $52500 \mathrm{~g}$. que constituían $69 \%$ de los sobrevivientes de BPN. Li peso de nacimiento fluctuó entre $900 \mathrm{y}$ 2 500 \& , ubicánduse la mayoria en el rango de $2000-2500 \mathrm{~g}$ (tabla 1). La edad gestacional varió critre 27 y $43 \mathrm{scm} a n a s$. siendo la mayoria pretérmino ai nacer (tabla 2), 53\% fueron pequeños para la edad gestacional (PEG) y $790_{0}$ presenlaton APGAK $\geq 7$ a los 5 minutos. El nivel sociocconómico. medido a través de la ficha CAS (Ministerio de Salud, Chilej se concentró en los índices más bajos 4 y 5 (72\%). Cuarenta y nueve (60\%) eran de sexo femenino. Fn el momento de la evaluación la edad de los niños nuctuaba cntre 7 años 10 meses y 8 años 8 meses.

Ll grupo control estuvo compuesto por igual núrncro de niños scleccionados entre los nacidos vivos en el mismo hospital y año, cuyo peso de nacimiento fue $\geq 2501 \mathrm{~g}$, paruados con el grupo de estudio en las variables de nivel suciocconómico y sexo. Fntre cllos, la edad gestacional fluctuó entre 36 y 43 semanas, siendo $91 \%$ de tétrino (1abla 2), $72 \%$ adecuados y $24 \%$ pequeños para la edad gesiacional. Sus edades fluctuaron entre 8 años y 8 años 11 moses.

L a húsqueda de los niños se hizo en base al registro de partos del hospital Juan Nó́ de Arica, del año calendario 1980, completándose la infomación con la revisión de las fichas clínicas de la madre y del recićn nacido. Iin todos los niños se reviś́ Ja edad gestacional consignadia en al tibro de panos a partir del registro de la fecha de ja última menstnación. Para la calificación de la adecuación del peso de nacimento con la edad gestacional se utilizó la gráfica de crecimiento fetal de Vacye y Dixon ${ }^{15}$, clasificación que scparó a los niños en adecuados (entre los percentiles 10 y 90). pequeños (percentil inferior a 10) o grandes para la edad gestacional (percentil mayor a 90). En la selección del grup' control se considerú que la distribución de scxo fuesc homugénea cn ambos grupos. Una vez que se corroboraron los domicilios, se entrevistó a las madres con la finalidad de parear ambus grupos en téminos del nivel sociucconómico, utilizando para ello la ficha CAS.

Ambos grupos fueron cvaluados en aspectos específicos del aprendizaje escolar por una profesora de educación diferencial. Se cnvio a los profesores de curso una pauka, la

\section{Tabla 1}

Distribución de niños del grupo de cstudio según peso de nacimiento

\begin{tabular}{|c|c|c|}
\hline $\begin{array}{l}\text { Peso de nacimiento } \\
\text { (g) }\end{array}$ & n & $\%$ \\
\hline$\leq 1000$ & 2 & 2,4 \\
\hline $1001-1499$ & 4 & 4,8 \\
\hline $1500-1999$ & 21 & 25,6 \\
\hline $2000-2500$ & 55 & 67,2 \\
\hline Total 82 & 00,0 & \\
\hline
\end{tabular}

Tabla 2

Distribución de los riños de los grupos estudio y control según cdad gestacional

\begin{tabular}{|c|c|c|c|c|}
\hline \multirow[t]{2}{*}{ Semanas gestación } & \multicolumn{2}{|c|}{ Grupo estudio } & \multicolumn{2}{|c|}{ Grupo control } \\
\hline & n & $\%$ & $\mathbf{n}$ & $\%$ \\
\hline$\leq 27$ & 1 & 1,2 & & \\
\hline $28 \cdot 30$ & 5 & 6,1 & & \\
\hline $31-33$ & 23 & 28,1 & & \\
\hline $34-36$ & 20 & 24,4 & 5 & 6,1 \\
\hline $37-42$ & 31 & 37,8 & 75 & 91.5 \\
\hline$\geq 43$ & 2 & 2,4 & 2 & 2,4 \\
\hline Total & 82 & 100,0 & 82 & 100,0 \\
\hline
\end{tabular}

que al ser contestada permitió conocer el promedio de notas del último año cursado por el niño.

El rendimiento escolar fue desglosado cn seis indicadores. Fi primero fuc el icndimiento escolas general, rcpresentado por la nota final promedjo del último año cursado. El segundo fue el nivel de lectura, evaluado a trayés del rendimicnto en la prucba exploratoria de dislexia cspecífica PEDE'6, la cual permite determinar el nivel del lector sobrc la base de lecura en oumplejidad crccicnte. Eista prucba está estandarizada para la población chilena y sc expresa en percentiles y puntajes " $\mathrm{t}$ " de la nomia. En esta ocasión se utilizó el puntaje "t" comparando el descmpeño del niño con respecto al nivel de su curso. La presencia de ertorcs en lectura fue el tercer indicador, evaluado a través del puntaje oblenido por el uiño en la subprueba "crrores específicos" dc la prucba exploraturia de dislexia específica PEDE y raducido a puntaje " $\mathrm{f}$ " según curso. El cuarto fue la comprensión de lectura, medida a través de la prucba de comprensión lectora de complejj dad lingüistica progresiva CLP ${ }^{17}$, estandarizada para la población chilcria. El quinto criterio, nivel de escritura fuc craluado mediante dictado de palabras y oraciones y redacción esprontánea de un tcma dado, extraído de la prucha de leclura y escritura del doctor $\mathrm{O} \mathbf{c a}^{\mathrm{I}}{ }^{\mathrm{k}}$. Jijnalmente, se midió la integración visomutora empleando la prueba de desarrollo de la integración visomotora Bccry ${ }^{19}$, la cual permite obtencr una edad de desarrollo visomotor. Para hacer comparables los resultados, considerando que la edad del total de los niños fluctuaba entre 7 años 10 meses y 8 años 11 meses, se obtuvo un coeficiente dividiendo la edad de integración visomotora por la edad cronológica del nit̃o $y$ multiplicando el resultado por 100.

l. a edad gestacional se describió como pretérnino $(\leq 36$ semanas) y témnino ( 237 semanas). La aducuación del peso de nacimiento con la edad gestacional se consideró adecuada cuando era igual o mayor que el percentil 10 y se calificó de pequeño al niñu que estaba bajo el percentil 10 .

Se aplicó un arálisis de varianza de dos factores en cada una de las variables de rendimicnto escolar, relacionando peso de nacimierio, edad gestacional y adecuación del peso a la edad gestacional. Se aceptó un nivel de significación igual o menor de 0,05. 


\section{Resultados}

El peso de nacimiento y la cdad gestacional tuvicron efectos significativos sobre algunos aspectos cspecíficos de aprendizaje escolar. Según se puede apreciar en la tabla 3, cl grupo de niños de bajo pcso rindió significativamentc más bajo en las pruebas que evalúan nivel de lectura, presencia de errores espccílicos en la lectura y comprensión lectora, al compararlos con los niños cuyo peso fue mayor a $2500 \mathrm{~g}$. A su vez, los niños prematuros rindieron significativamente menos en las pruebas que miden nivel de lectura y presencia de errores específicos en la lectura (cabla 4). Sin embargo, en relación a ta adecuación a la edad gestacional, sólo se observó diferencia en la prucba de integración visomotora. en la cual el grupo pequeño para la edad gestacional tuvo un rendimiento significativamente mayor que el grupo adecuado a la edad gestacional (tabla 5). No se observaron efectos de interacción entre las variables peso de nacimiento, edad gestacional y adccuación a la edad gestacional.

En cl grupo de bajo peso se detectaron cuato niños con limitaciones severas: dos con deficiencia mental leve, uno con parálisis cerebral, uno con parălisis cerebral y deficiencia mental moderada. Asimismo, en cl grupo de peso mayor a $2500 \mathrm{~g}$ se delectaron cuatro niños con dificultades severas, que se relacionaban con deficiencia mental y problemas de audición y lenguaje. Considerando que estos ocho niños, que obluvicron puntajes mínjmos en las pruebas aplicadas, podían sesgar los resultados, se realizaron los análisis de varianza incluyêndolos en la mucstra y posteriormente excluyéndolos. Al climinarlos, adquirió también significación el efecto favorable del mayor peso de nacimiento sobre el nivel de cscritura, mantenićndose todos los otros hallazgos sin modificaciones.

\section{Comentario}

Los resuliados enconirados en este esiludio son concordantes con la literatura revisada, en el sentido de que tanto el bajo peso de nacimiento como la premature $z$ afectan negativamente cicrtos aspectos específicos del aprendizaje escolar, tales como aquellos relacionados con la lectoescritura ${ }^{3,6}, 7,10-14$. Sin cmbargo, estos defectos no
T'abla 3

Rendimicnto escolitr según peso de nacimiento, excluidos cuatro niños con déficjt mental en cada grupo

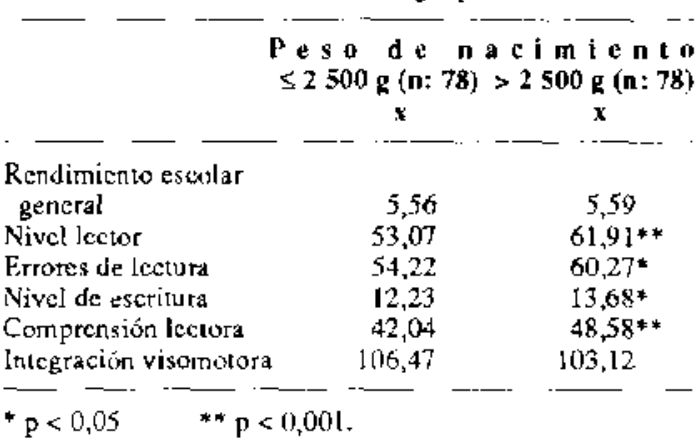

Tabla 4

Rendimiento escolar según edad gestacionai, excluidos 8 ninos con deficiencia ruental

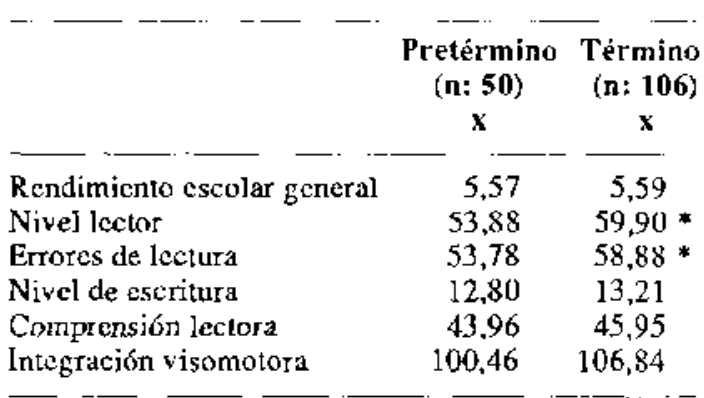

$* p<0,05$.

fueron detectados en el rendimicnto escolar general ponderado mediante la nota final promedio del úllimo año cursado, que constituye un indicador más grueso, que no daria cuenta de dificullades más cspecílicas.

Es posibie que en el presente trabajo las altcraciones del rendimiento escolar sean más discretas, porque los niños que pesaron menos de $1500 \mathrm{~g}$ al nacer, tuvieron una representación menor que la esperada en la muestra; $0,1 \%$ en ve $\%$ de $0,7-1 \%$, proporción disminuida debido al fallecimiento de 26 niños de muy bajo peso de nacimiento en el período neonital.

En cste estudio se controló el efecto del nivel socioeconómico sobre el aprendizaje cscolar, sin embargo otros estudios revelan que los niños 


\section{Tabla 5}

Rendimiento escolar según adecuación del peso a la cdad gestacional, excluidos 8 niños con deficiencia mental

\begin{tabular}{lrr} 
& $\begin{array}{c}\text { PEG } \\
(\mathbf{n : 5 9})\end{array}$ & $\begin{array}{r}\text { A } \mathbf{5 G} \\
(\mathbf{n} ; \mathbf{9 7})\end{array}$ \\
\hline Rendimiento escolar gencral & 5,55 & 5,59 \\
Nivel lector & 56,17 & 58,29 \\
Errores de lectura & 55,93 & 58,04 \\
Nivel de escritura & 12,88 & 13,19 \\
Comprension lectora & 43,31 & 45,92 \\
Integración visomotora & $111,49 *$ & 100,72 \\
\hline
\end{tabular}

$* p<0.05$.

provenientes de bajo nivel socioeconómico prescntan mayor incidencia de dificultad de aprendizaje que aquellos procedentes dc cstratos sociales más altos ${ }^{20}$. Cabe entonces suponer que aquellos niños nacidos prematuros y, o, de bajo peso y que pertenecen a un nivel socioeconómico bajo presentaran un riesgo mayor de dificultad en su rendimiento escolar.

$\mathrm{Si}$ a este hecho se agrega el antecedente revelado en otro estudio nacional, respecto a que lat dificuliad presentada para aprender a leer y escribir en los primeros años de escolaridad va asociado a una alta probabilidad de presentar bajo rendimiento escolar en años posteriores ${ }^{21}$ sc podría suponer que este grupo esté expuesto a un mayor riesgo de deserción escolar, lo cual acectará su calidad de vida en la edad adulta. al limitar sus expectativas de inserción laboral.

Con refcrencia a la mayor representación de mujeres en la muestra, si se considera el antece. dente de que los problemas de aprendizaje de la lectoescritura se dan con mayor frecuencia cn los niños varones, podríamos afirmar que cn este grupo, dada la mayor proporción de niñas, las diferencias cncontradas en las variables de aprendizaje escolar cstudiadas son menos evidentes que las que resultarian de tener una distribución similar de varones y niñas en el grupo de estudio.

Habría sido de interés conocer el nivel intelectual del grupo de niños estudiado, para así poder deicrminar en qué medida las diferencias encontradas en el aprendizaje de la lectoescritura se originan en una capacidad general de aprendizajc más limitada.

Sin embargo, pese a sus limitaciones, este estudio aporta información que pucde contribuir al conocimiento de las secuclas que afectan a los nifios nacidos de bajo peso y se suma a olras publicaciones nacionales que buscan idenificar factores de riesgo para cl aprendizaje es$\operatorname{colar}^{22-24}$.

\section{Resumen}

Se estudió la influencia que cjercen el peso de nacimiento, la cdad gestacional y la adecuación del peso a la edad gestacional, sobre algunas variables del aprendizaje escolar en 82 niños nacidos de bajo peso en el Hospital Regional de Arica, en $1980 \mathrm{c}$ igual número de niños con peso superior a $2500 \mathrm{~g}$, pareando ambos grupos en términos de nivel sociocconómico y sexo. Después de excluir cuatro niños de cada grupo por defíciencia mental, los niños de bajo peso (n: 78) tuvieron un rendimicnto significativamente inferior en las calificaciones promedio de sus niveles de lectura $(53,07$ vs $61,91, p<0,001)$, escritura $(12,23$ vs $13,68, p<0,05)$, errores dc lectura $(54,22$ vs $60,27, \mathrm{p}<0,05)$ y comprensión lectora $(42,04$ vs $48,58, p<0,001)$ que el grupo de peso mayor a $2500 \mathrm{~g}$ ( $\mathrm{n}: 78$ ). Los niños prematuros (n: 50) tuvieron un rendimiento significativamente menor en nivel de lectura $(53,88$ vs $59,90, p<0,05)$ y errores de lectura $(53,78$ vs $58,88, \mathrm{p}<0,05)$, que los nacidos de término (n: 106). Los pequetios para la edad gestacional (n: 59) presentaron mayor desarrollo de integración visomotora $(11,49$ vs $100,72, \mathrm{p}<0,05)$ que el grupo de peso adecuado. Los resultados apoyan la necesidad de preparar programas cducativos preventivos para estos grupos de riesgo.

(Palabras clave: aprendizaje, peso al nacer.)

\section{Agradecimientos}

Los autores agradecen al Director del Hospital Dr. Juan Yóe de Arica, Dr. Oscar Tortealba, quien autonizó la revísión de fistitas médicas de los niños del estudio y al Sr. Patricio Durán. Jefe del Scrvicio Municipal de Educación de Arica, por dar amplias facilidades para ubicar a los niños en los colegios. 


\section{Referencias}

1. Torres-Pereyre J: Emphasis on l'reventive Pcrinatolo. gy: a suttablc altemative for develuping countrics. Semin Perinatol 1988; 12: 435-440.

2. Goldenberg $R$, Nelson $K$, Ilale $C$, Wayne J, Barolucei $A$, Koski $J$ : Survival of infants with low birhweight and carly gestational age, 1979 to 1981. An J Obstel Gynccol 1984; 149: 523.531.

3. Hirata T, Epcar J, Walsh A et al.: Survival and outcome of infanls 501 to $750 \mathrm{gm}$ : six-year cxperience. J l'cdiatr 1983: 102: 741-748.

4. Rooth $G$ : Low birthweight and neurological scquelac A summary of some swedish studies. Jin Kubli Ji, Patel $N$, Schmidt $W$. Perinatal events and brain damage in surbiving children, Berlin: Springer-Verlag. 1988.

5. Hun! J, Tooley W, Marvin D: Learning disabilitics in children with birth weights $51500 \mathrm{~g}$. Semin Perinatol 1982; 6: 280-287.

6. Kitchen $W$, Ryan $M$, Richards $A$, et al.: $\Lambda$ longitudinal study of very low-birhweight infants IV: $A$ tr uvervicw of perfomance at cight years of age. Dev Mcd Chil Neurol 1980; 22: 172-188.

7. Noble-Jamieson C, Lukeman D, Silverman As, Davie. $P$ : Low birhweight children at school age: neurological, psychological and pulmonary function. Semin Perinatol 1982; 6: 266-273.

8. Driscoll J.Jr, Driscoll $J$, Steir $M$, ex al.: Mortality and morbidity in infants Iess than 1.001 grans birth wcight. Pedialrics 1982; 69: 21-24.

9. Fitzhardinge PM: [iollowing-up studies on the low bjrthweight infant. Clin Perinatol 1976; 3: 503 515.

10. Neligan G, Kolvin I. Scott D, Garside R: Bom to seon or bom to small. A follow up study to seven years of agc. Clinics Dev Med 1976; 61: 19-37.

11. Drillien $C$, Thomson A, Burgoine $K$ : Low birthwejght children at carly school-age: A longitudinal study. Dev Med Child Seurol 1980; 22: 26-47.

12. Calame A, Fawer C, Clalys V, Arrazola L, Ducret $S$, Jaunin $L$ : Neuto developmental outcome and school purfomance of very-low birthweight infants at eight ycars of age. Eur J Pcdiatr 1986; 145: 461-466.

13. Eilers B, Deasai $N$, Wilson $M$, Cunningham $M$ : Classroom performance and social factors of children with birtheeghts of 1.250 grams or Jess: Follow-up at 5 to years of age. Pcdiatrics 1986; 77: 203-208.

14. Roberison $C_{\text {s }}$ Etches P. Kyle J: Eighl year school performance and growth of preterm, small for gestational agc. J Jediat 1990; 116: 19-26.

15. Naeye R, Dixon J: Distortions in fetal growth slandards. Pedialr Res 1978; 12: 987-991.

16. Berdicewski $O$, Milicic $N$, Orellana $E$ : Llaboración de normas para la prueba de dislexia específica de Condemarín-Blomquist. Documento $\mathrm{N}^{5} 47$, Facultad de Fdueación, Pontificia Universidad Católica de Chile, $1987,2^{2}$ edición.

17. Alliende $F$, Condemarín M, Milicic N: Manual para la aplicación de la Prueba de Comprensión Lectura de Complejidad Lingǘśtica Progresiva $2^{4}$ cdición. Słgo. Ediciones Universidad Calólica de Chile. 1987.

18. Olea $R$ : Prucba de lectura y escritura. Fi Niño Limitado. $1979 ; 6: 4-9$

19. Beery $K$ : Development lest of Visual-Motor Integration. Chicago Follet, 1967.

20. BravoL, Monienegro H: Educación, niñez y pobreza. $1^{\text {a }}$ cdición. Santiago Ediciones Nucva Universidad. liditurial Universitaria S.A., 1977.

21. Manterola A, Avendaño $P$, Coironeo $f$, Avendaño $A$, Va!enzueda $C$ - Aprendizaje de la lectuescritura inicial como poder pronóstico del rendimicno escolar ulterior. Rev Chil Pediatr 1985; 56: 334-339.

22. Manterola $A$, Avendaño $A$, Valenzuela $C$, et al : Verificación de relacioncs entre pnucbas neurológicas y ucndimiento escolar. Rev Chil Pediatr 1983: 54: 20-24.

23. Manterolo A, Avendaño $P$. Cotroneo $Y$, Avendaño A, Valenzuela $C$ : Factores dc niesgo en las dificultades dc aprendizaje escolar en niños de medio cconómics social medio y bajo. Rev Chil Pediatr 1986;57: $318-$ 325.

24. Manterola A, Avendaño A, Valenzula $C$. Avendaño $P$. Cotroneo Y: Examen neurologico y rendimiento cscohar: correlaciones a siele años plazo. Rev Chil Pediatr 1989; 60: 157-165. 